\title{
LA PRESENCIA DE LA MUJER EN EL SECTOR TURÍSTICO DE GALICIA
}

THE PRESENCE OF WOMEN IN THE TOURISM SECTOR IN GALICIA

\section{Jakson Renner Rodrigues Soares}

Doutor em Direção e Planificação do Turismo pela Universidade da Coruña - UDC, (Espanha). Professor pela Universidade de Santiago de Compostela - USC e pela Universidade da Coruña - UDC. E-mail: jaksonrenner@yahoo.com.br

\section{Larissa Paola Macedo Castro Gabriel}

Doutora pela Universidade da Coruña - UDC, (Espanha). Pesquisadora pela Universidade da Coruña - UDC. E-mail: larissa.gabriel@udc.es

\section{Raquel Santiago Romo}

Mestranda pela Universidade da Coruña - UDC, (Espanha).E-mail: raquelsromo@gmail.com 


\title{
LA PRESENCIA DE LA MUJER EN EL SECTOR TURÍSTICO DE GALICIA
}

\section{RESUMEN}

Al observar el equipo executivo de cualquiera empresa gallega, muy probablemente nos daremos cuenta que de una pequeña presencia femenina. Aun habiendo años de esfuerzos para cambiar esa realidad, todavía se percibe que buena parte del liderazgo empresarial está en manos de hombres. Aunque se puede concordar que sería posible que más mujeres alcanzasen altas posiciones jerárquicas, ese cambio es lento, necesita acciones osadas y cabría esperar que no fuese necesario tanto tempo hasta que la representación de ellas en puestos de mandos sea tan considerable a nivel de igualarse a la representación masculina. El sistema de género, constituido por los valores, normas, significados, roles, estereotipos, sanciones..., deja lugar a una organización social jerarquizada, en la que los puestos clave de poder están ocupados mayoritariamente por hombres. De esta manera, se bien es cierto, que ya encontramos un acercamiento al tema en la existencia de estudios del rol desempeñado por la mujer en turismo, la mayoría de ellos tratan concretamente de una parte del sector. Por lo tanto, hace interesante investigar de manera más general y que englobe todo el sector, sin olvidarse la importancia de conocer datos que permitan una comparativa entre formación y empoderamiento en la gestión.

Palabras Clave: Género; Gestión del turismo; Ambiente empresarial; Liderado femenino. Galicia.

\section{THE PRESENCE OF WOMEN IN THE TOURISM SECTOR IN GALICIA}

\begin{abstract}
Observing the executive team of any Galician company, we probably will find a small female presence. Even having years of efforts to change that reality, it is still perceived that most of the business leadership is in the hands of men. Although we can agree that it would be possible that more women reach high hierarchical positions, we have to assume that this change is slow and need bold actions and it should not be necessary wait until that the representation of females in positions of command be so considerable equated to male representation. The gender system, consisting of the values, norms, meanings, roles, stereotypes, sanctions..., generates in a hierarchical social organization, in which the positions of power are occupied mostly by men. Thus, although we already an approach to the subject in studies on the role played by women in tourism, most of them deal specifically a part of the sector. Therefore, it becomes interesting to investigate in more general terms the whole sector, not forgetting the importance of knowing data which allow to perform a comparison between professional training and empowerment in management.
\end{abstract}

Keywords: Gender; Tourism Management; Business Environment; Female Leadership, Galicia. 


\section{INTRODUCCIÓN}

El turismo en España supone uno de los pilares de la economía constituyendo el sector que presenta un mayor aumento en la economía. Este hecho queda reflejado en el avance del PIB turístico que registró una cifra del 3,7\% (Exceltur, 2015). Sin embargo, el sector sigue siendo caracterizado por una importante desprofesionalizacion y relevante desigualdad de género en cuanto a salario y puestos de responsabilidad (OMT, 2011; Albacete \& Fuentes, 2010; Hernández et al., 2011).

Dentro del escenario turístico español, Galicia adquiere un protagonismo de gran índole arrojando cifras turísticas de una enorme competitividad. Este hecho se constata a través del Informe de Conxuntura Turística de Xullo de 2016, en el cual se observa que la región ocupa el séptimo lugar en el volumen de demanda dentro del conjunto del Estado. De este modo, se registra un aumento con una cifra total de 203.00 nuevas pernoctaciones en establecimientos reglados. Concretamente en este año, coincidiendo con el último Año Xacobeo, se alcanza un máximo histórico con cerca de 600.000 viajeros y algo más de 1.2 millones de pernoctaciones sólo en el mes de julio.

Para logar el objetivo de este trabajo se realizó un análisis de la situación del turismo profesional en Galicia, teniendo en cuenta los datos del sector público y privado, recogidos a través de fuentes primarias y secundarias. Por lo tanto, este artículo, abarca una labor cuantitativa descriptiva con el fin de obtener un diagnóstico de dicho sector en Galicia desde una perspectiva de género.

\section{LA PRESENCIA DE LA MUJER EN LA FORMACIÓN TURÍSTICA}

En líneas generales, según el Global Report On Women In Tourism (2011), el turismo puede suponer una de las herramientas para el empoderamiento de la mujer, de hecho a través del informe anteriormente citado, se pretende hallar aquellos indicadores que permitan monitorizar el rol que desempeña la mujer en este sector y así constituir las metas a conseguir. En este mismo sentido, se debe considerar que las empresas apoyen la igualdad entre géneros, visto que ella es también un indicativo más de que la organización respecta principios de responsabilidad social (Calvo Gallego, 2010).

En cuanto a la presencia de estudios universitarios en el campo del turismo, ofertados por las diferentes universidades gallegas, Universidade da Coruña (UDC), Universidade de Revista de Gestão e Secretariado-GeSec, São Paulo, v. 8, n. 1, p 26-47,jan./abr. 2017. 
Santiago (USC) y Universidade de Vigo (UVIGO), se asegura el interés de realizar un estudio relativo a la industria turística en Galicia. No obstante, el tema principal que se aborda, dada la relevancia de los factores que se exponen anteriormente, se centra en la posición de la mujer en esta actividad en Galicia.

Uno de los motivos principales por los que se genera la investigación surge tras la observación de la amplia presencia de la mujer en los estudios universitarios de turismo en esta región. Concretamente, suponiendo en muchos casos un número superior al de alumnos del género masculino. Así mismo, en el año 2013, en todos los cursos de Máster ofertados por las universidades gallegas en materia de turismo, las mujeres componen cuantitativamente la mayor parte del alumnado matriculado.

Con todo, los datos proporcionados por la Consellería de Educación de la Xunta (2013), indican que el Máster Universitario en Dirección y Planificación del Turismo de Interior y de Salud (UVIGO) contaba con un total de 35 alumnos matriculados, de los cuales 26 eran mujeres. Además, el Máster Universitario en Planificación y Gestión de Destinos y Nuevos Productos Turísticos (UDC), las mujeres componían aproximadamente el $71 \%$ del total del alumnado matriculado. Por último en la USC, específicamente en el Máster Universitario en Turismo Urbano y Gestión de Empresas Turísticas, la presencia femenina se traduce en 25 mujeres frente a un total de 30 alumnos matriculados. No obstante, supone solo una pequeña muestra de la presencia femenina en las universidades gallegas, datos que se desarrollarán a lo largo del estudio. De este modo, resulta obvio destacar que, en Galicia y en uno de los últimos escalones en cuanto a formación en el sector, la mujer ocupa un mayor porcentaje en relación a la presencia masculina.

La conjunción de todos los factores citados anteriormente generan la siguiente cuestión, si la presencia de la mujer en la universidad a nivel cuantitativo resulta mayor, surge la necesidad de conocer en qué medida se refleja el papel de la misma en los puestos de liderazgo a todas las escalas de empleo en el sector. Fue pensando en esta cuestión que a través de este trabajo se hizo una diagnosis del sector turístico gallego.

\section{METODOLOGÍA}

Tomando como base los pasos necesarios para alcanzar la profesionalización, realizamos una investigación exploratoria, descriptiva y de campo Bilert y Biscoli (2011). Eso porque no se hizo un trabajo de este tipo con antelación a este. Además, como bien destacan 
las autoras, una investigación como esta se desarrolla a partir de la descripción de datos primarios. En primer lugar, se realizó un análisis descriptivo de la situación académica del ámbito del turismo en Galicia, con el objetivo de tener una referencia de la presencia de hombres y mujeres cualificados en el sector. En concreto, lo datos para este primer análisis descriptivo se obtuvieron a partir del número de inscritos en los cursos de grado, master y doctorado de turismo de las Universidades de A Coruña, Santiago de Compostela y Vigo.

En segundo lugar, con el fin de analizar la situación profesional, se realizó una revisión descriptiva estadística de la presencia de hombres y mujeres en el mercado turístico gallego, además se ha tenido en cuenta tanto el sector público como el privado, identificando el género de los altos cargos de algunas de las principales empresas y organismos públicos representativos del sector.

Para el análisis del sector público a nivel gallego se identificaron los más altos cargos de la Agencia de Turismo de Galicia (Turgalicia) y sus departamentos. Este órgano tiene como objetivo impulsar, coordinar y gestionar la política autonómica en materia de turismo y, en especial, la promoción y la ordenación del turismo dentro de la comunidad (Xunta de Galicia, 2012). Se puso en evidencia la situación de otros organismos dependientes de la misma y de la Consejería Gallega de Turismo. Por medio de este análisis se ha podido revisar la presencia de hombres y mujeres en los puestos de responsabilidad de la región.

En relación al sector privado, en primer lugar se realizó un análisis a partir del ranking de Hosteltur de cadenas hoteleras de 2015. De dicho documento se seleccionaron 4 cadenas del ranking de las 10 más importantes en relación al nivel de facturación, presentes en los tres principales centros urbanos gallegos (A Coruña, Santiago de Compostela y Vigo). Para completar el estudio se eligió 1 hotel de cada cadena por ciudad y se realizaron contactos telefónicos con el fin de averiguar el género del principal responsable de cada uno de los hoteles. Las cadenas seleccionadas fueron: Meliá, NH Hoteles, Hotusa y AC Hoteles. En total 10 hoteles fueron contactados y no se obtuvo respuesta solo de 1 hotel de todo el listado.

En un segundo momento fueron contactados los principales hoteles de 1, 2, 3, 4 y 5 estrellas de las ciudades de A Coruña y Santiago de Compostela, con el objetivo de comparar la situación de la mujer en los cargos de responsabilidad de hoteles de las dos ciudades mencionadas. En total fueron contactados 20 hoteles de A Coruña y 45 hoteles de Santiago de Compostela.

En cuanto a las agencias de viajes, a partir del ranking publicado por Hosteltur de Agencias de viajes (2016), se elaboró un listado seleccionando 4 de las 5 grandes redes en Revista de Gestão e Secretariado -GeSec, São Paulo, v. 8, n. 1, p 26-47,jan./abr. 2017. 
relación al nivel de facturación presentes en las ciudades gallegas de A Coruña, Santiago de Compostela y Vigo. Una agencia de cada rede por ciudad ha sido contactada telefónicamente, con el fin de indagar el género del responsable por la oficina. En total, 10 agencias fueron contactadas: Viajes el Corte Inglés, Viajes Halcon-Ecuador, Barceló Viajes y Nautalia.

Este análisis nos permite tener una visión clara de la situación del sector turístico en Galicia a nivel profesional y proponer una radiografía de la representatividad de las mujeres a nivel profesional en el ámbito turístico gallego. Así como plantear una serie de líneas estratégicas capaces, por lo menos, de mejorar la situación de desigualdad económica y social entre hombres y mujeres.

\section{LA SITUACIÓN DE LAS MUJERES EN EL SISTEMA UNIVERSITARIO DE} GALICIA

En este apartado se llevará a cabo un análisis general de la participación de la presencia de la mujer en el Sistema Universitario Gallego (SUG), en concreto se estudia la demanda de formación en cursos de grado, $1^{\mathrm{er}}$ y $2^{\circ}$ ciclo, y postgrados. Posteriormente, se analiza de manera más específica el perfil de la demanda del estudiantado de los cursos universitarios de turismo impartidos por las universidades de A Coruña, Vigo y Santiago de Compostela. Salvagni y Canabarro (2015) recuerdan que aunque la lucha de las mujeres por romper con los paradigmas, estudiando y formándose más, desarrollando mejor sus habilidades y aptitudes, aún no es suficiente para combatir a la desigualdad de género en el mercado de trabajo.

De acuerdo con los datos del Sistema Universitario Español (SUE), en el curso 20142015 Galicia contó con un total de 3 Universidades, estructuradas en 7 campus y 6 sedes universitarios. En el curso 2013-2014 se han matriculado en las universidades gallegas, en estudios de grado y máster 64.538 estudiantes. Del total de estudiantes, 46.699 son de grado, 12.320 de primer y segundo ciclo y 5.519 de máster. Según los indicadores de acceso al Grado en universidades públicas presenciales por comunidad autónoma, en el curso 20132014 el Sistema Universitario Gallego ofreció un total de 120 grados, sumando un total de 11.847 nuevas plazas, de las cuales $94,5 \%$ fueron ocupadas.

En términos globales, el 34,5\% de los estudiantes se matricularon en las titulaciones pertenecientes a la rama de ingeniería y arquitectura, el 33,3\% en ciencias sociales y jurídicas, el $14,5 \%$ ciencias, el $14,5 \%$ artes y humanidades, y solo $10,9 \%$ en ciencias de la salud. El 
siguiente gráfico ilustra la distribución de los estudiantes en las universidades de Galicia por rama de enseñanza, curso 2013-2014.

Gráfico 1: Porcentaje de matriculados en Galicia por rama de estudios

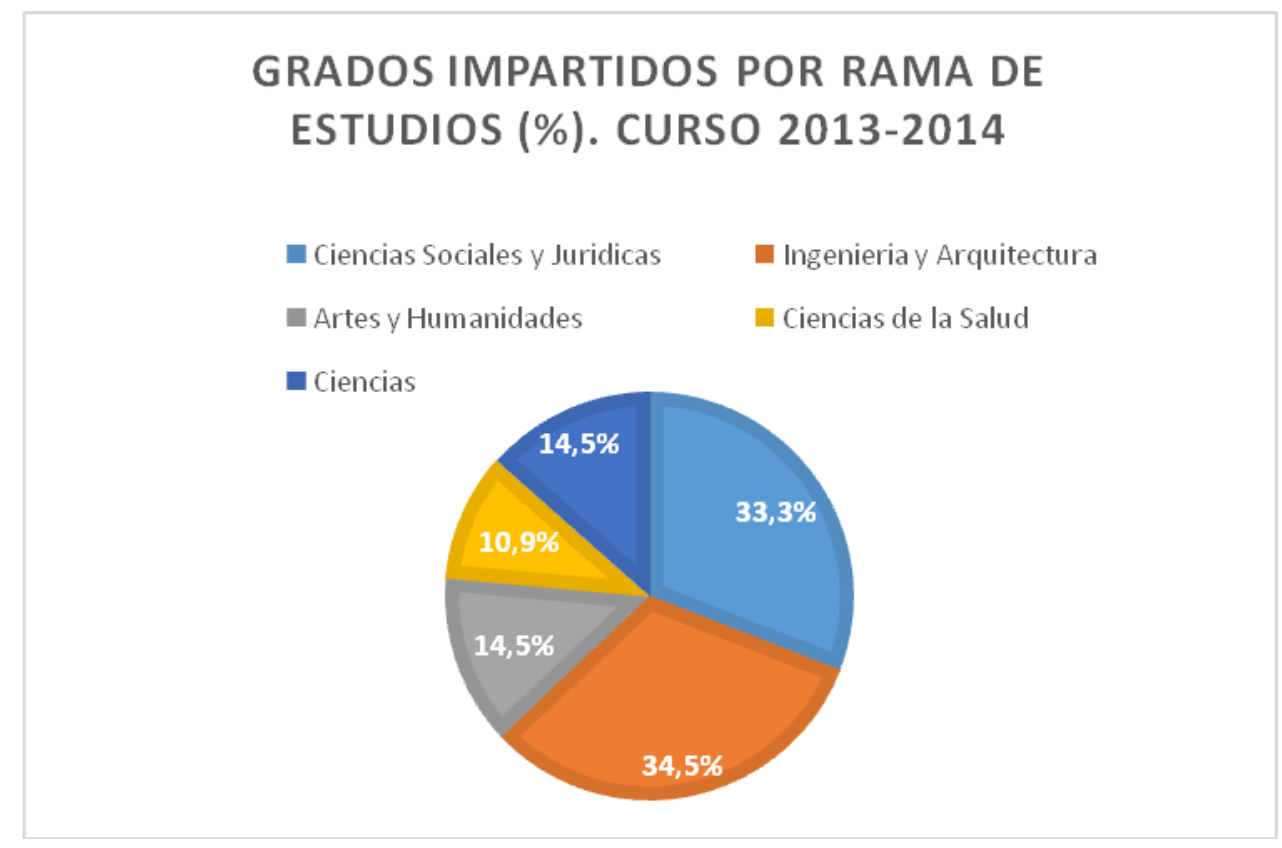

Fuente: Sistema Universitario Español - SUE (2015)

Sin embargo la tasa de variación decenal de los estudiantes matriculados en las universidades públicas, en los cursos de Grado y $1^{\text {er }}$ y $2^{\circ}$ ciclo, cursos 2003-2004 y 20132014 es uno de los peores de España con un -30\%. Considerando los datos del sistema universitario nacional, que demuestran un aumento de la presencia de las universidades privadas en España, probablemente estos datos ponen de manifiesto el aumento de la cuota de mercado de estas universidades en Galicia, disminuyendo la búsqueda por universidades públicas.

En el curso 2014-2015 se impartieron 180 másteres en las universidades públicas gallegas sumando un total de 5.415 plazas ofertadas. En el análisis por rama se observa que la tendencia nacional puede ser atribuida a la región de Galicia en relación a las matrículas en los cursos de grado, puesto que las plazas ofertadas en la rama de ciencias sociales y jurídicas representan el $47,4 \%$, el $22,9 \%$ en la de ingeniería y arquitectura, cerca del $9 \%$ en artes y humanidades, $9 \%$ en ciencias de la salud y el $11,2 \%$ en ciencias.

De acuerdo con el SUE, en el curso 2013-2014, el 54,4\% de los estudiantes matriculados en las universidades públicas son mujeres, sin embargo este número sube entre la población egresada representando un total de 57,3\%. Los datos son muy similares de los Revista de Gestão e Secretariado-GeSec, São Paulo, v. 8, n. 1, p 26-47,jan./abr. 2017. 
años precedentes, donde también se observa una proporción de hombres superior a la de mujeres solo en la rama de ingeniería y arquitectura. Sin embargo la distribución se invierte en la rama de ciencias de la salud, rama en la que las mujeres tienen una presencia superior a la de hombres. Esto se traduce en el porcentaje que alcanza la presencia femenina en esta rama abarcando un $69,7 \%$. La distribución por rama también se presenta similar entre los estudiantes de master, el 53,7\% lo componen mujeres. Asimismo, en España 10.889 tesis doctorales fueron leídas en el año 2013, de las que 5.361 fueron leídas por mujeres. El 21,4\% por mujeres en un rango de edad comprendida entre los 24 y 29 años, el 38, 6\% por mujeres de 30 a 34 años, el 17\% por mujeres de 35 a 39 años y el 23\% por mujeres de 40 a 55 años.

Siguiendo esta misma tendencia, según los datos del Instituto Galego de Estadística (IGE) referente al curso 2013-2014, en el caso específico de Galicia, del total de estudiantes matriculados en los grados, $1^{\text {er }}$ y $2^{\circ}$ ciclo, el 55,2\% lo componen mujeres. Con relación a los datos nacionales, en Galicia se repite la tendencia nacional, una proporción de hombres superior solo en la rama de ingeniería y arquitectura. El siguiente gráfico ilustra la presencia de las mujeres matriculadas en las universidades gallegas durante el curso 2013-2014 por rama de enseñanza.

Gráfico 2: Porcentaje de mujeres matriculadas en las universidades gallegas por rama de estudio 2013-2014

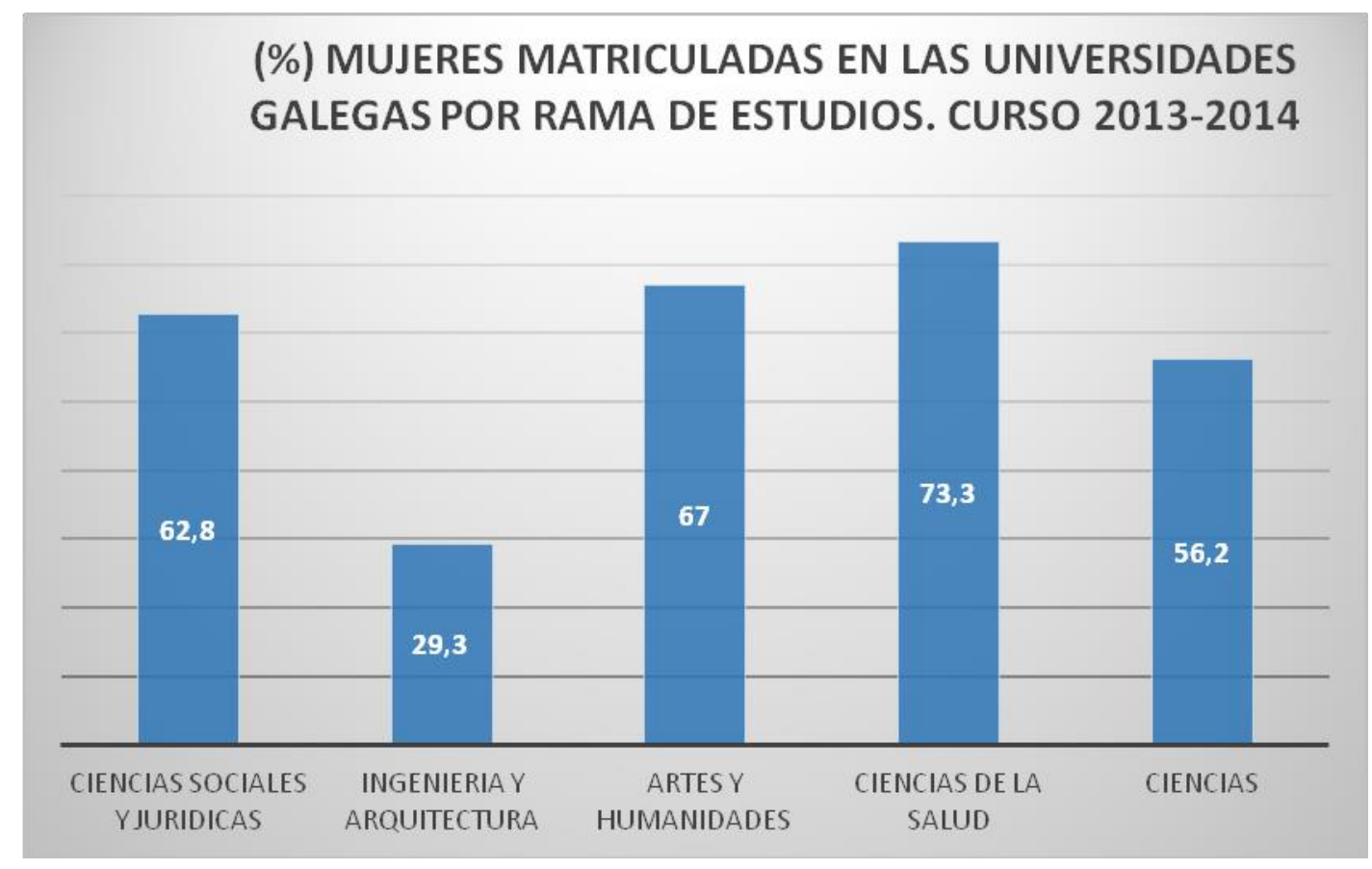

Fuente: Instituto Galego de Estadística -IGE 
En relación a los másteres, de acuerdo con el SUG, en el curso 2012-2013 del total de 4.893 estudiantes matriculados, el $57 \%$ son mujeres. Además fueron leídas un total de 550 tesis doctorales en el año 2013, y el 53\% de ellas fueron leídas por mujeres.

A partir de este análisis cuantitativo de las estadísticas generales, se deduce que no existen diferencias entre géneros en el acceso al sistema educativo gallego, sin embargo se observa que los estereotipos continúan condicionando la elección de los estudiantes. En este sentido, como bien puntúan Barros, Izequiel y Silva (2011) en sus estudios sobre la equidad de género del profesional de secretariado, las características del perfil profesional tienden a cambiar la visión estereotipada relativo a la profesión, pero solo serán extintas cuando se llegue a la igualdad entre los géneros.

Resulta destacable la presencia privilegiada de las mujeres en el sistema universitario, suponiendo un porcentaje superior al $50 \%$ de las matrículas. No obstante, cuando analizamos los gráficos de la distribución por rama de estudio, encontramos lo mismo que Carvalho (2016), donde a día de hoy aún se encuentra una clara separación de géneros en la educación superior. Los datos apuntan a una distribución estereotipada por género, es decir, todavía la presencia de hombres en los cursos de las ramas de ingeniería y arquitectura es muy superior en comparación con las mujeres, mientras que la situación se invierte cuando analizamos los datos referentes a las ramas de ciencias de salud, indicando una mayor presencia de estas. Para Basanta (2015), continúa la tendencia de la presencia de la mujer en las carreras dedicadas a los cuidados contrastando con la escasa presencia de ellas en las carreras consideradas masculinas.

En relación a las carreras direccionadas a la formación universitaria en el ámbito del turismo, las matrículas efectuadas en el curso 2013-2014 fueron distribuidas de la siguiente manera: 
Tabla 1: Alumnos matriculados en los cursos de turismo de las universidades galegas

\begin{tabular}{|c|c|}
\hline Curso Universitario UDC & $\mathrm{N}^{\circ}$ Matriculas \\
\hline Grado en Turismo & 183 \\
\hline Grado en Ciencias Empresariales / Grado en Turismo & 33 \\
\hline Diplomado en Turismo & 40 \\
\hline Máster Universitario en Dirección y Planificación del Turismo & 9 \\
\hline Curso Universitario UVIGO & $\mathrm{N}^{\circ}$ Matriculas \\
\hline Diplomado en Turismo & 14 \\
\hline Grado en Turismo & 230 \\
\hline Máster Universitario en Dirección y Planificación del Turismo & 19 \\
\hline Máster Universitario en Dirección y Planificación del Turismo Interior y de Salud & 35 \\
\hline Curso Universitario USC & $\mathrm{N}^{\circ}$ Matriculas \\
\hline Máster Universitario en Turismo Urbano y Gestión de Empresas & 30 \\
\hline
\end{tabular}

Fuente: SUG (2013)

Aunque el informe publicado por el SUG (2012-2013) no indique las cifras representativas de la presencia femenina en los cursos de turismo, los datos referentes a la rama de enseñanza ciencias sociales por sexo, mencionados anteriormente nos llevan a deducir que la mayoría de las matrículas fueron realizadas por mujeres, siguiendo de este modo la tendencia nacional.

A la luz de este breve análisis estadístico descriptivo de los datos publicados por el SUG y la presencia de la mujer en la legislación, y tras la lectura de la Ley gallega 7/2004 para la igualdad de mujeres y hombres, del 16 de julio, que incorpora aspectos como mayor presencia de las mujeres en los nos contenidos del currículo, no admisión de contenidos que transmitan estereotipos sexistas, etc., se considera que el momento en que nos encontramos existe un cierto interés por el fomento una política de igualdad de género. Sin embargo, numerosos estudios demostraron que todavía existe una desigualdad de acceso al conocimiento adecuado, el que acaba por generar una no igualdad en el trato y evidencian que en el sistema de educación se generan y se mantienen los estereotipos y discriminaciones sociales (Castro \& Reimóndez, 2013; Basanta, 2015).

\section{LAS MUJERES EN EL SECTOR TURÍSTICO GALLEGO}

El turismo se ha convertido en España en la fuente principal de desarrollo económico desde la década de los sesenta, aportando en torno a un 10,9\% al PIB nacional. Los puestos de trabajo asociados a este sector representaron el 12,7\% del total de los empleos de la economía Revista de Gestão e Secretariado-GeSec, São Paulo, v. 8, n. 1, p 26-47,jan./abr. 2017. 
española en 2014, según los datos publicados por el Instituto Nacional de Estadística (INE, 2016). Sin embargo, los datos publicados por la Organización Mundial del Turismo (OMT, 2011) indican que existe una significativa desigualdad de género en relación a salario y posiciones de responsabilidad. El Informe Global de la Mujer de la OMT/ONU sobre la Mujer de Turismo muestra que el turismo puede ofrecer importantes oportunidades para reducir la brecha de género en el empleo y desarrollar el espíritu empresarial en las mujeres, pues que éste sector ofrece el doble de probabilidades para que se ellas conviertan en empleadores en turismo, en comparación con otros sectores.

El Informe Global Report on Women in Tourism (2011) muestra que, en cuanto a su presencia en servicios y oficinas, las mujeres están bien representadas. Pero hay una escasa presencia en puestos de trabajo a niveles profesional y además ganan de $10 \%$ a $15 \%$ menos que sus homólogos masculinos. En este sentido, los gobiernos y el sector privado tienen un papel importante en el desempeño de la promoción de políticas para intensificar la igualdad y el empoderamiento de la mujer. Asimismo, como bien destacan Sánchez-Fernández, VacaAcosta y Vargas-Sánchez (2016), promover la incorporación de la mujer en cargos de mandos en las empresas es una acción socialmente responsable. No solo eso, así se puede tener un impacto positivo en la gestión, dado que ellas están más formadas, tienen conocimientos basados en la diversidad y tienen más sensibilidad con la responsabilidad social, por ejemplo.

En España, el salario medio anual de 2012 fue de 22.726,40 euros, aunque el sueldo más frecuente se sitúa en torno a 15.500 euros anuales. Las mujeres cobran solo un $76,1 \%$ del salario de los hombres. Además, son diversos los estudios que evidencian que el número de mujeres en puestos directivos es desproporcionadamente bajo, y ponen de manifiesto la existencia de barreras para que la mujer acceda a puestos de liderazgo y responsabilidad. Incluso cuando mujeres y hombres han demostrado ser igual de eficientes a la hora de asumir responsabilidades de dirección (Mooney \& Ryan, 2009; Marco-Lajara \& Úbeda-García, 2013; Villamil \& Alonso, 2013; Heuete, Brotons \& Sigüenza, 2016).

De acuerdo con los datos del INE (2013), las mujeres representan el 60\% de los empleados en el sector del turismo, y su presencia en puestos de responsabilidad en los ámbitos públicos y privados es muy inferior a los puestos ocupados por hombres (Heuete, Brotons \& Sigüenza, 2016). De acuerdo con el Anual de Empleo en el Sector Turístico, en España, en 2012, el porcentaje de mujeres trabajando en el sector turístico tiene una clara tendencia descendente, puesto que ellas representaban un 48,2\% en 2008 , mientras que en 2012 llegaban a un 45,4\%. Además, las mujeres que trabajan en turismo están, en promedio, Revista de Gestão e Secretariado -GeSec, São Paulo, v. 8, n. 1, p 26-47,jan./abr. 2017. 
mejor formadas que los hombres. Igualmente, el informe evidencia que hay menos mujeres con contrato indefinido ( $70 \%$ versus $64 \%$ ) y, la tasa de temporalidad es superior en el caso de las mujeres (35\% versus 29\%).

Pasando al tema en el que se centra nuestra investigación, el ámbito profesional del sector en Galicia, hemos analizado los datos publicados por la Xunta de Galicia, en el Informe anual 2015 sobre el Empleo en el sector turístico gallego. De acuerdo con el informe, el número de personas ocupadas en el sector en este año es de 108.813, el que representa una variación de $-2,7 \%$ respecto al año de 2014. El peso de los ocupados en el sector representa un $10 \%$ del total de ocupados en Galicia. La tasa de paro en 2015 para el sector turístico fue de $13 \%, 2 \%$ menor que la tasa de paro de 2014. Sin embargo, cuando se analiza el peso relativo de la economía galega frente a economía española, se evidencia que el peso en la economía galega se ha reducido tanto en términos turísticos como para el conjunto de la economía de la comunidad autónoma.

De acuerdo con los datos del Informe Empleo en el sector turístico gallego año 2015, en el conjunto de ocupados relacionado con las ramas características del turismo, del total de 108.813 personas ocupadas en el sector, los subsectores que con mayor ocupación es el de servicios de comidas y bebidas, con un 52,8\%. Teniendo en cuenta el género, el 53,1\% de los ocupados en el sector son mujeres, lo que implica que se trata de un sector predominantemente feminizado. El grupo de ocupados con estudios superiores representa el $29,0 \%$ en 2015.

La presencia por género en las ramas características del turismo es la siguiente:

- Transporte de viajeros: el 74,7 \% de los ocupados son hombres, que representan el $13,0 \%$ del total de ocupados en el sector turístico;

- Servicios de alojamiento: el 56,8 \% de los ocupados son mujeres, que representan o 4,2\% del total de ocupados en el sector turístico;

- Servicios de comidas y bebidas: en esta rama el 62,1 \% son mujeres, que representan el 32,8 \% del total de ocupados ene 1 sector del turismo.

En relación a la distribución por género atribuidas a cargos de más alta responsabilidad en el ámbito profesional del sector en Galicia, hemos analizado por separado el nivel público y privado:

\section{$\underline{\text { Sector Público }}$}

A nivel autonómico se consideraron los cuatro organismos gubernamentales más importantes del sector. Así, la distribución por género atribuidas a cargos de más alta responsabilidad puede ser representada de la siguiente manera:

Revista de Gestão e Secretariado-GeSec, São Paulo, v. 8, n. 1, p 26-47,jan./abr. 2017. 
La Presencia de La Mujer en El Sector Turístico de Galicia

Tabla 2: Distribución por género de los responsables públicos

\begin{tabular}{|l|c|}
\hline \multicolumn{1}{|c|}{ Entidad } & Genero del Director General \\
\hline Agencia Turismo de Galicia & Mujer \\
\hline S.A. de Xestión do Plan Xacobeo & Hombre \\
\hline Consejo Galego de Turismo & Mujer \\
\hline Área de Estudos e Investigación de Turismo de Galicia & Hombre \\
\hline
\end{tabular}

Fuente:Elaboración propia

Debido a que la Agencia de Turismo de Galicia es la agencia pública autonómica, que tiene como objetivo impulsar, coordinar y gestionar la política autonómica en materia de turismo, con la ayuda de las demás entidades citadas en la tabla anterior, dado el papel importante y actividades desarrolladas por estas entidades se considera interesante para este análisis evidenciar el género del puesto de responsabilidad relacionado. En este caso encontramos la igualdad de género, aunque a nivel autonómico, la gestión en materia de turismo, en algunos casos coexisten varios organismos y en otros existe sólo uno, por lo que el número de responsables puede variar en cada comunidad (Berrozpe, Lasarte \& Martinez, 2014).

La siguiente tabla enumera los puestos de responsabilidad ocupados por hombres y mujeres al interno de cada entidad. Estos datos representan el género de las principales funciones que conforman en organigrama de cada entidad. En el caso de la Agencia de Turismo de Galicia, cabe destacar que se trata de un dato aproximativo, puesto que no ha sido posible encontrar datos relativos a la dirección de Recursos Humanos y de los responsables por áreas provinciales.

Tabla 3: Puestos de responsabilidad ocupados por hombres y mujeres según entidad

\begin{tabular}{|l|c|c|}
\hline \multirow{2}{*}{\multicolumn{1}{|c|}{ Entidad }} & \multicolumn{2}{c|}{ Género de los puestos de responsabilidad } \\
\cline { 2 - 3 } & Hombres & Mujeres \\
\hline Agencia Turismo de Galicia & 6 & 9 \\
\hline S.A. de Xestión do Plan Xacobeo & 4 & 0 \\
\hline Consejo Galego de Turismo & 18 & 8 \\
\hline
\end{tabular}

Fuente: Elaboración propia

Asimismo, cabe destacar que la tabla 3 incluye los datos referentes al Consejo Gallego de Turismo, que se trata de un órgano de asesoramiento, apoyo y propuesta para los asuntos referidos a la ordenación, promoción, fomento y desarrollo del turismo de Galicia. Representa el género de sus miembros responsables por las áreas, los consejeros de turismo en líneas Revista de Gestão e Secretariado-GeSec, São Paulo, v. 8, n. 1, p 26-47,jan./abr. 2017. 
generales son formados por alcaldes, representantes de sindicatos, de universidades y representantes de las empresas del sector turístico gallego. Como se puede ver, la presencia de mujeres en los cargos de responsabilidad de la agencia de turismo es ligeramente superior a la presencia de los hombres, sin embargo, en los demás casos analizados, contrariamente, la presencia de los hombres es elevadamente superior si comparada con la presencia de las mujeres.

\section{$\underline{\text { Sector Privado }}$}

En cuanto a la diagnosis del sector privado, en los dos primeros casos se ha tenido en cuenta para el análisis las principales cadenas hoteleras y agencias de viajes españolas con oficinas presentes en Galicia, de acuerdo con su nivel de facturación en el año 2015, según Hosteltur (2016), como ya se ha mencionado anteriormente. El resultado de ambos casos se resume en las siguientes tablas:

Tabla 4: Distribución por género de los responsables de los hoteles pertenecientes de las principales cadenas presentes en Galicia

\begin{tabular}{|l|l|l|}
\hline \multicolumn{3}{|c|}{ A Coruña } \\
\hline Meliá & María pita & Hombre \\
\hline NH hotel - Hotel Hesperia & Hotel Hesperia A Coruña & Hombre \\
\hline Hotusa - Eurostars & Eurostars & Mujer \\
\hline AC Hotels & Ac Hotel & Hombre \\
\hline \multicolumn{2}{|c|}{ Santiago de Compostela } & \\
\hline Meliá & Tryp & Hombre \\
\hline NH hotel - Hotel Hesperia & Hesperia Peregrino & Hombre \\
\hline Hotusa - Eurostars & Eurostar & Hombre \\
\hline \multicolumn{2}{|c|}{ Vigo } & \\
\hline Meliá & Tryp Vigo los Galeones Hotel & Hombre \\
\hline NH hotel & Hesperia Vigo & / \\
\hline Hotusa - Eurostars & Eurostar & Mujer \\
\hline AC Hotels & AC Palacio Hotel & Hombre \\
\hline Total hombres: & & $\mathbf{8}$ hombres \\
\hline Total mujeres: & Fuente: Elaboración propia & \\
\hline
\end{tabular}

Como se puede ver en la tabla 4, salvo la particularidad de los hoteles Eurostar-A Coruña y Vigo, del total de 11 hoteles contactados pertenecientes a las principales cadenas de hoteles presentes en Galicia, solo 2 de ellos son dirigidos por mujeres. No ha sido posible 
obtener información en relación a la gestión del Hotel Hesperia de Vigo, debido a problemas de transferencia de llamada desde la central de atendimiento de Madrid hacia el hotel de Vigo.

En cuanto a las agencias de viajes encontramos la siguiente tabla:

Tabla 5: Distribución por género de los responsables de las oficinas pertenecientes a las principales agencias de viajes

\begin{tabular}{|c|c|c|}
\hline \multicolumn{3}{|c|}{ A Coruña } \\
\hline Cadena & Ubicación & Responsable \\
\hline Viajes el Corte Ingles & Coruña-Barcelona & Hombre \\
\hline Viajes Halcón-Ecuador & Federico Tapia & Hombre \\
\hline Barceló Viajes & Plaza de la Galera & Mujer \\
\hline Nautalia & Juan Flores & Hombre \\
\hline \multicolumn{3}{|c|}{ Santiago de Compostela } \\
\hline Cadena & Dirección & Responsable \\
\hline Viajes el Corte Ingles & Rosalía de Castro & Mujer \\
\hline Viajes Halcon-Ecuador & Carrera do Conde & Hombre \\
\hline Barceló Viajes & Xeneral Pardiñas & Hombre \\
\hline Nautalia & Horreo & Mujer \\
\hline \multicolumn{3}{|c|}{ Vigo } \\
\hline Cadena & Dirección & Responsable \\
\hline Viajes el Corte Ingles & C.C. Vigo & Mujer \\
\hline Viajes Halcón-Ecuador & García Barbón & Mujer \\
\hline Barceló Viajes & Av. Del Alcalde Gregorio Espino & Hombre \\
\hline Nautalia & Gran Vía & Mujer \\
\hline Total hombres: & \multicolumn{2}{|l|}{6 hombres } \\
\hline Total mujeres: & \multicolumn{2}{|l|}{6 mujeres } \\
\hline
\end{tabular}

Fuente: Elaboración propia

Puesto que algunas de las tres ciudades estudiadas cuentan con más de una oficina de la misma red de agencias de viajes, las oficinas contactadas fueron seleccionadas de manera aleatoria. A partir de la tabla 5 se evidencia que la situación relativa a los cargos de responsabilidad es distinta con relación a la gestión de los hoteles de las principales cadenas presentes en Galicia. Se identifica un equilibrio de representatividad de género en los cargos de responsabilidad de las oficinas. De un total de 12 agencias contactas, 6 oficinas son gestionadas por mujeres, es decir, una situación paritaria.

En cuanto a la situación general de los hoteles presentes en A Coruña y Santiago de Compostela, para completar el análisis fueron contactados aleatoriamente hoteles de 1, 2, 3, 4 Revista de Gestão e Secretariado-GeSec, São Paulo, v. 8, n. 1, p 26-47,jan./abr. 2017. 
e 5 estrellas con el fin de comprobar la distribución por género en los puestos de responsabilidad.

Tabla 6: Situación general de los hoteles presentes en A Coruña

\begin{tabular}{|c|c|c|c|c|c|}
\hline \multicolumn{6}{|c|}{ A Coruña } \\
\hline \multirow{3}{*}{ Mujer } & Hotel 1* & Hotel 2* & Hotel 3* & Hotel 4* & \multirow{3}{*}{ l } \\
\hline & Almirante & Santa Catalina & I & Zenit & \\
\hline & $\begin{array}{l}\text { Francisco } \\
\text { Javier }\end{array}$ & l & l & l & \\
\hline \multirow{7}{*}{ Hombre } & Hotel 1* & Hotel $2 *$ & Hotel 3* & Hotel 4* & Hotel $5 *$ \\
\hline & Castiñeiras & Maycar & Plaza & $\mathrm{AC}$ & $\begin{array}{l}\text { Hesperia } \\
\text { Finisterre }\end{array}$ \\
\hline & Coruñamar & Moon & Riazor & Attica 21 & l \\
\hline & Cristal & l & l & Trip & l \\
\hline & Los Lagos & / & l & Eurostars & l \\
\hline & Mar del Plata & l & I & $\begin{array}{c}\text { Meliá } \\
\text { María Pita }\end{array}$ & l \\
\hline & / & l & I & $\begin{array}{c}\mathrm{NH} \\
\text { Atlántico }\end{array}$ & I \\
\hline Total hombres: & \multicolumn{5}{|c|}{16 hombres } \\
\hline Total mujeres: & \multicolumn{5}{|c|}{5 mujeres } \\
\hline
\end{tabular}

Fuente: Elaboración propia

A partir de los datos presentados en la Tabla 6, de los 20 hoteles contactados, solo 5 hoteles tienen como responsable mujeres. Del mismo modo la tabla 7 abajo, demuestra que la situación en Santiago no es distinta, del total de 44 hoteles contactados, solo 15 de ellos tienen mujeres como responsable principal. 
La Presencia de La Mujer en El Sector Turístico de Galicia

Tabla 7: Situación general de los hoteles presentes en Santiago de Compostela

\begin{tabular}{|c|c|c|c|c|c|}
\hline \multicolumn{6}{|c|}{ Santiago de Compostela } \\
\hline \multirow{8}{*}{ Mujer } & Hotel 1* & Hotel 2* & Hotel 3* & Hotel 4* & \multirow{8}{*}{ / } \\
\hline & B Noir & Santa Lucía & $\begin{array}{c}\text { Ciudad de } \\
\text { Compostela }\end{array}$ & $\begin{array}{l}\text { Hotel A quinta } \\
\text { da Auga }\end{array}$ & \\
\hline & $\begin{array}{c}\text { Fonte de } \\
\text { San Roque }\end{array}$ & Montenegro & $\begin{array}{l}\text { Carris Casa } \\
\text { de la Troya }\end{array}$ & Compostela & \\
\hline & San Jacobo & Moure & $\begin{array}{l}\text { Santiago } \\
\text { Apóstol }\end{array}$ & I & \\
\hline & I & I & San Carlos & I & \\
\hline & I & l & Rúa Villar & I & \\
\hline & I & & $\begin{array}{c}\text { Hotel } \\
\text { Altamira }\end{array}$ & I & \\
\hline & l & l & Gelmírez & l & \\
\hline \multirow{9}{*}{ Hombre } & Hotel 1* & Hotel 2* & Hotel 3* & Hotel 4* & Hotel 5* \\
\hline & $\begin{array}{l}\text { Capital de } \\
\text { Galicia }\end{array}$ & San Clemente & Herradura & $\begin{array}{l}\text { Eurostars San } \\
\text { Lázaro }\end{array}$ & $\begin{array}{c}\text { Eurostars } \\
\text { Araguaney }\end{array}$ \\
\hline & $\begin{array}{c}\text { Casa de } \\
\text { Amancio }\end{array}$ & Punta Cana & $\begin{array}{l}\text { Next Style } \\
\text { Santiago de } \\
\text { Compostela }\end{array}$ & $\begin{array}{c}\text { Gran Hotel } \\
\text { Santiago }\end{array}$ & NH Obradoiro \\
\hline & Santa Clara & Pazos Alba & Pombal & $\begin{array}{l}\text { Hesperia } \\
\text { Peregrino }\end{array}$ & $\begin{array}{c}\text { Palacio del } \\
\text { Carmen }\end{array}$ \\
\hline & Real B\&B & $\begin{array}{l}\text { Miradoiro } \\
\text { Belvis }\end{array}$ & Ruta Jacobea & Los Abetos & $\begin{array}{c}\text { Parador Hostal } \\
\text { Dos Reis } \\
\text { Católicos }\end{array}$ \\
\hline & I & $\begin{array}{l}\text { A Tafona do } \\
\text { Peregrino }\end{array}$ & San Lorenzo & $\begin{array}{l}\text { Puerta del } \\
\text { Camino }\end{array}$ & l \\
\hline & I & Hórreo & San Miguel & $\begin{array}{c}\text { Torres de } \\
\text { Compostela }\end{array}$ & I \\
\hline & I & Entrecercas & I & Virxe da Cerca & I \\
\hline & l & Costa Vella & / & / & / \\
\hline Total hombres: & \multicolumn{5}{|c|}{29 hombres } \\
\hline Total mujeres: & \multicolumn{5}{|c|}{15 mujeres } \\
\hline
\end{tabular}

Fuente: Elaboración propia

A partir de estos datos podríamos decir que de un modo en general, los altos cargos de los principales hoteles en Galicia están ostentados por hombres. Quizás este diagnóstico se 
trate de un reflejo de las políticas de igualdad de género, adoptadas por el gobierno que no exige una presencia mínima en los puestos de responsabilidad de las empresas privadas. Asimismo, como bien destacó Menezes, Oliveira y Diniz (2013) en sus estudios sobre mujeres ejecutivas, si por un lado se considera que la mujer tiene múltiples competencias y capacidad de dividirse entre distintas tareas, por otro, eses atributos contribuyen para aumentar los prejuicios contra ellas. Con todo, se esperan más trabajos, y más profundos, acerca de por qué aún existe esa brecha entre hombres y mujeres en el sector turístico.

\section{CONCLUSIONES}

Como vemos, a pesar de existencia de leyes para promover la igualdad entre hombres y mujeres, y de la fuerte presencia femenina en los cursos universitarios, los datos estadísticos todavía apuntan a la existencia de estereotipos respecto a los tipos de carrera universitaria y el género de sus matriculados. Con todo, cabe destacar que la Universidad no puede ser considerada la causa de esta diferenciación, sin embargo, debería ser un motor para fomentar el cambio necesario.

En el ámbito profesional del turismo, la desigualdad de género parece ser una constante, lo que acaba afectando no solo a la rentabilidad social, pero también la economía y la calidad del sector OMT (2011). Además, las empresas privadas y las entidades públicas acaban por perder un recurso muy valioso, que son mujeres con alta formación, al no darles oportunidad para demostrar su capacidad y talento profesionales (Canalis, 2011). Además, no se puede olvidar que las instancias gubernamentales pueden definir pautas de la actuación empresarial donde una de ellas es la estableciendo estándares mínimos en el ámbito laboral, buscando por ejemplo, la igualdad de género y medidas de conciliación laboral y personal (Melle Hernández, 2007).

Como hemos visto durante el proceso de presentación y análisis de los datos, Galicia forma anualmente una gran cantidad de mujeres y aún así, ese número no repercute en la dirección de las empresas. La universidad forma profesionales para asumir cargos de liderazgo. Es decir, el rango más alto de las empresas. Aunque el número de profesionales femeninas formadas en turismo es muy superior a lo de hombres, como hemos notado, en la realidad empresarial esa relación no se respecta. Por lo tanto, al igual que Nogueira y Kubo (2013) destacaron en sus estudios sobre el liderazgo femenino en los puestos de mando en la empresa brasileña, en Galicia también es necesario discutir la inserción laboral de la mujer en 
el mercado laboral, más específicamente en el sector turístico. Eso porque no solo se puede desperdiciar las potenciales directivas formadas para el sector, pero tampoco se puede obviar su alto nivel de competencia y capacidades.

Quizás hacen falta políticas con directivas objetivas que buscan una igualdad de género incorporada de manera efectiva al sector, y capaces de conviértela en un importante factor de cohesión social y que puede favorecer el turismo en esta doble vertiente social y económica (Lugo et al., 2011).

Se debe considerar la limitación de este trabajo en no reflexionar los discursos y las opiniones de las ejecutivas sobre la cuestión de la equidad en el sector turístico gallego. Pero cabe destacar que esta primera etapa del estudio que se presentan aquí en este artículo solo objetivaba explorar el campo y analizar las evidencias ofertadas por los datos primarios. De ahí entonces que resultan nuestras recomendaciones y futuras líneas de actuación, que es considerar, como informantes, a las mujeres que actúan como mandos en las empresas turísticas gallegas. Así, se podrá llegar a los impedimentos que ellas mismas atribuyen al desarrollo de sus carreras profesionales en el sector.

\section{REFERENCIAS}

Albacete, C. A. \& Fuentes, M. M. (2010). Difusión de la Investigación Española Sobre Turismo en Revistas Internacionales. Revista de Análisis Turístico, vol. 9, pp.14-29.

Barros, C. M. P., Izequiel, D. S. A. \& Silva, J. S. (2011). Os desafios enfrentados pelo profissional de secretariado executivo do gênero masculino nas organizações contemporâneas, Revista de Gestão e Secretariado, vol. 2, n. 1, pp. 158-176.

Basanta, N. (2015). Evolución histórica das mulleres no ensino: Da educación femenina á coeducación en igualdade. EDUGA Revista Galega do Ensino, $\mathrm{n}^{\circ} 70$ / xullo-decembro 2015 .

Bilert,V. S. \& Biscoli, F. V. (2011). Perfil dos discentes (ingressantes e concluintes) de secretariado executivo: um estudo comparativo nas instituições de ensino superior (IES) públicas, Revista de Gestão e Secretariado, vol. 2, n. 2, pp. 33-57. 
Calvo Gallego, F. J. (2010). Responsabilidad social y relaciones laborales. Temas Laborales, 104, pp. 41-83.

Carvalho, R. O. (2016). Sociedade, mulher e profissão. Revista de Gestão e Secretariado, vol. 7, n. 1, pp. 27-44.

Castro, O. \& Reimóndez, M. (2013). Feminismos. Vigo: Edicións Xerais de Galicia.

Canalis, X. (2011). Mujer y turismo: la igualdad no existe [Versión electrónica]. Hosteltur, 208, pp. 6-15.

Consellería de Educación de la Xunta. (2013). Datos estadísticos do sistema universitario de Galicia, 2012-2013.

Lugo, G., Alberti, M.P., Figueroa, O.L. \& Talavera, D. (2011). Patrimonio cultural y género como estrategia de desarrollo. Estado de México. En Pasos, vol. 9, no 4, pp. 599-612.

Hernández, J.M., Folgado, J.A. \& Campón, A.M. (2011). La investigación en turismo en España: una revisión de la literatura a través de las revistas de dirección de empresas. En IV Jornadas de Investigación en Turismo, pp. 100-120. Sevilla: 19 y 20 de mayo de 2011.

Huete, R., Brotons, M. \& Sigüenza, M. C. (2016). La desigualdad entre mujeres y hombres en el sector hostelero español. Estudios y Perspectivas en Turismo, vol. 25, pp. 7387.

Hinojosa, V. (2015). Ranking HOSTELTUR de cadenas hoteleras 2015. [Versión electrónica]. Hosteltur. Recuperado en 01 de agosto de 2016, de http://www.hosteltur.com/112791_ranking-hosteltur-cadenas-hoteleras-2015.html.

Instituto Galego de Estadística (IGE). Estudantes matriculados nas universidades galegas segundo rama de coñecemento e sexo. Datos referentes al curso 2013-2014. $\begin{array}{llllll}\text { Recuperado en } & \text { de } & \text { agosto de }\end{array}$ http://www.ige.eu/igebdt/selector.jsp?COD=2273\&paxina $=001 \& \mathrm{c}=0203002$ 
Instituto Nacional de Estadística (INE). (2013). Estadísticas sobre el mercado laboral. Madrid: INE.

Marco-Lajara, B. \& Úbeda-García, M. (2013). Human resource management approaches in Spanish hotels: An introductory analysis. International Journal of Hospitality Management, vol. 35, pp. 339-347.

Melle Hernández, M. (2007). La responsabilidad social dentro del sector público. Ekonomiaz, vol. 65, n.2, pp. 84-107.

Menezes, R. S. S., Oliveira, J. L. \& Diniz, A. P. R. (2013). Simbolismos de gênero e gestão: uma análise das feminilidades de executivas brasileiras, Revista de Gestão e Secretariado, vol. 4, n. 1, pp. 01-22.

Mooney, S. \& Ryan, I. (2009). A woman's place in hotel management: upstairs or downstairs? Gender in Management: An International Journal, vol. 24,n. 3, pp. 195-210.

Nogueira, E. C. O. R. \& Kubo, E. K. M. (2013). Sentidos do exercício da liderança por mulheres executivas brasileiras, Revista de Gestão e Secretariado, vol. 4, n. 2, pp. 114-133.

Organización Mundial del Turismo. (2011). Global Report on Women in Tourism. UNESCO.

Rosa, J. M. (2016). Ranking HOSTELTUR de Agencias de Viajes. [Versión electrónica]. Hosteltur. Recuperado en 01 de septiembre de 2016, de http://www.hosteltur.com/116250_ranking-hosteltur-agencias-viajes.html

Salvagni; J. \& Canabarro, J. (2015). Mulheres líderes: as desigualdades de gênero, carreira e família nas organizações de trabalho. Revista de Gestão e Secretariado, vol. 6, n. 2, pp. 88-110. 
Sánchez-Fernández, M.D., Vaca-Acosta, R. M. \& Vargas-Sánchez, A. (2016). Socially Responsible Practices in Hotels: A Gender Perspective. In Kaur, L. \& Ahmad, S. Corporate Social Responsibility in the Hospitality and Tourism Industry (pp. 28-45). Hershey PA: IGI Global.

Soria, J. M. (2015). El balance anual del turismo español en 10 claves. [Versión electrónica]. Hosteltur. Recuperado en 01 de septiembre de 2016, de http://www.hosteltur.com/114156_balance-anual-turismo-espanol-10-claves.html

SUE Ministerio de Educación, Cultura e Deporte (2015). Datos y cifras del sistema universitario español curso 2014-2015.

SUE Ministerio de Educación, Cultura e Deporte (2014). Datos y cifras del sistema universitario español curso 2013-2014

Turgalicia. (2016). Conxuntura turística en Galicia. AEI-TG: Area de estudos e investigación. Recuperado en 6 de septiembre de 2016, de http://www.turismo.gal/aei/portal/index.php?idm=163

World Tourism Organization (UNWTO) and the United Nations Entity for Gender Equality and the Empowerment of Women (UN Women) (2011). Global Report on Women in Tourism 2010.

Villamil, M. \& Alonso, M. M. (2013). Posición de las mujeres en los equipos de dirección y el crecimiento de las cadenas hoteleras a nivel mundial. Estudios Turísticos, n. 198, pp. 35-60.

Xunta de Galícia (2012). Decreto 196/2012, do 27 de setembro, polo que se crea a Axencia de Turismo de Galicia e se aproban os seus estatutos. 